\title{
Reliability of Thyroid Imaging Reporting and Data System (TIRADS) Classification in Differentiating Benign from Malignant Thyroid Nodules
}

\author{
Boniface Moifo $^{1 \#}$, Emmanuel Oben Takoeta ${ }^{1,2}$, Joshua Tambe ${ }^{1}$, François Blanc ${ }^{2}$, \\ Joseph Gonsu Fotsin ${ }^{1}$ \\ ${ }^{1}$ Department of Radiology and Radiation Oncology, Faculty of Medicine and Biomedical Sciences, \\ The University of Yaoundé I, Yaoundé, Cameroon \\ ${ }^{2}$ Centre Hospitalier de Lagny, Marne La Vallée, France \\ Email: "bmoifo@yahoo.fr
}

Received July 2, 2013; revised August 2, 2013; accepted August 9, 2013

Copyright (C) 2013 Boniface Moifo et al. This is an open access article distributed under the Creative Commons Attribution License, which permits unrestricted use, distribution, and reproduction in any medium, provided the original work is properly cited.

\begin{abstract}
Background: Ultrasonography (US) is the best diagnostic tool in the initial assessment of thyroid nodule. Giving its appropriateness and accessibility, ultrasound-based thyroid imaging reporting and data systems (TIRADS) classifications have been developed with main goal to standardize reporting and facilitate communication between practitioners, and to indicate when fine-needle aspiration biopsy (FNAB) should be performed. Objective: To determine the reliability of Russ' modified TIRADS classification in predicting thyroid malignancy. Materials and Methods: It was a cross sectional study carried out at Centre Hospitalier de Lagny, Marne La Vallée (France). Consecutive records of patients with focal thyroid nodules on ultrasound (US) for which US-guided FNAB was performed and pathology results were available, from January 2007 to August 2012, were selected for review. The risk of malignancy of each TIRADS category was determined and correlation with pathology assessed. Statistical performances of some US features were also assessed. The threshold for statistical significance was set at 0.05 . Results: A total of 430 records of patients were eligible. Twenty-three out of 430 (5.3\%) nodules were malignant. The risk of malignancy of the TIRADS categories were as follows: TIRADS $2 \%$, TIRADS 3 2.2\%, TIRADS $_{4 \mathrm{~A}} 5.9 \%$, $^{2}$ IRADS $_{4 \mathrm{~B}} 57.9 \%$, TIRADS $_{5} 100 \%$ (Gamma statistic $=0.85$; Spearman correlation $=0.30$, Pearson's $R=0.37, \mathrm{p}<0.001$ ). Some US features were associated with a higher risk of malignancy: irregular contours $(\mathrm{OR}=22.4)$, taller-than-wide shape $(\mathrm{OR}=19.5)$, microcalcifications $(\mathrm{OR}=15.2)$, and marked hypoechogenicity $(\mathrm{OR}=12.7)$. Conclusion: Russ' modified TIRADS classification is reliable in predicting thyroid malignancy. More evidence is nevertheless necessary for widespread adoption and use.
\end{abstract}

Keywords: TIRADS; Thyroid Nodule; Thyroid Cancer; Ultrasonography; Fine-Needle Biopsy

\section{Introduction}

Ultrasonography (US) is widely used in the assessment of the thyroid gland. Among the different pathologies that can be depicted and characterized by US are nodules. Nodules can be benign or malignant. Some studies have

\footnotetext{
${ }^{*}$ Competing interests: The authors declare that they have no competing interests.

Authors' contributions: BM conceived the study and participated in its design, review of the images, data collection and drafting of the manuscript. EOT participated in the study design, review of the images, data collection and statistical analysis. JT participated in the review of the images, statistical analysis and proof-reading of the manuscript. FB examined all the patients and participated in data collection. JGF participated in the study design and proofreading of the manuscript. All authors read and approved the final manuscript.

${ }^{\#}$ Corresponding author.
}

shown that less than $10 \%$ of thyroid nodules are malignant $[1,2]$ and that thyroid US depicts nodules in up to $50 \%$ to $67 \%$ of the population [3-5].

Some US-features are in favor of benignity or malignity, especially when grouped together. Suspicious nodules will require fine-needle aspiration biopsy (FNAB) for pathology analysis. When should FNAB be performed? Although some guidelines have been proposed [2,6-8], some confusion still exists as the same nodule may be classified differently using different guidelines implying different diagnostic or therapeutic attitudes.

The terminology "Thyroid Imaging Reporting and Data System” (TIRADS) was first used by Horvath et al. [9], drawing inspiration from the "Breast Imaging and 
Reporting Data System” (BIRADS) of the American College of Radiology [10]. This was in a bid to standardize the reporting of results of thyroid US that can be understood by clinicians and also stratify the risk of malignancy of a lesion based on the US features of the lesion. Horvath et al. described 10 US patterns of thyroid nodules and related the rate of malignancy according to the pattern [9]. However, these US patterns were not applicable to all thyroid nodules and appeared difficult to use in routine clinical practice. Park et al. [11] proposed an equation for predicting the probability of malignancy in thyroid nodules on the basis of 12 US features. Although this approach makes it possible to stratify nodules into categories, it can be difficult to assign every thyroid nodule into the equation proposed in clinical practice. To further achieve a practical tool in the hands of sonographers in analysing thyroid nodules and to improve communication between radiologists and clinicians, Russ et al. [12] proposed a TIRADS classification that was further modified after feedback from those who used it [13].

It is therefore apparent that a highly reliable, reproducible and clinically practical TIRADS classification will greatly improve communication between clinicians and radiologists. This will even be more helpful in settings where FNAB is not readily available and so decisions will therefore be based to a great extent on the US features of the lesions and TIRADS classification as this implies the potential risk for malignancy. It is against this background that this study was designed to assess the reliability of the modified TIRADS classification proposed by Russ et al. [13] in risk stratification for malignancy in a group of patients who had FNAB performed on some focal thyroid nodules.

\section{Materials and Methods}

It was a cross-sectional descriptive and analytical study carried out at Centre Hospitalier de Lagny, Marne La Vallée (France) from July 2012 to January 2013 with retrolective data collection. Authorization for the study was obtained from the local hospital authorities. Consecutive records of all patients from January 2007 to August 2012 with focal thyroid nodules on ultrasound (US) for which US-guided fine-needle aspiration biopsy (FNAB) was performed and pathology results were available were selected for review. All records without available digital thyroid US images and those with indeterminate cytology/histology were excluded.

\subsection{Imaging and Imaging Analysis [5]}

All US scans of the thyroid gland and neck areas were performed using a linear-array transducer (5 - $12 \mathrm{MHz}$ ) on a Philips US scanner (iU22 Philips Medical Systems, Bothell, Wash) using an optimized gain. One radiologist with more than ten years of experience performed all of the thyroid US scans.

All thyroid nodules were characterized according to the internal component (solid, mixed or cystic), the margins, echogenicity, evidence of calcifications and the shape. Margins were classified as well circumscribed, lobulated or irregular. Echogenicity was classified as "hyperechogenicity", "isoechogenicity", "hypoechogenicity" and "marked hypoechogenicity". Isoechogenicity was defined as an echogenicity similar to that of the adjacent healthy thyroid gland. A nodule was classified as "marked hypoechogenicity" if the echogenicity was less than that of the superficial surrounding neck muscles. When present, calcifications were categorized as micro-calcifications $(<3 \mathrm{~mm})$ and macrocalcifications $(>3 \mathrm{~mm}$ with acoustic shadowing). The shape of the nodule was categorized as "taller-than-wide" (greater in its antero-posterior dimension than in its transverse dimension) and "wider-than-tall".

Using the modified Russ classification [13], each nodule was classified into a TIRADS category (1, 2, 3, 4A, $4 \mathrm{~B}$ and 5) based on the US features.

\subsection{US-Guided FNAB}

After US evaluation of the thyroid gland, US-guided FNAB was performed by the same radiologist who performed the US scan. US-guided FNAB was performed with a 23-gauge needle attached to a $10 \mathrm{ml}$ disposable plastic syringe. Materials obtained from aspiration biopsy were expelled onto glass slides, smeared and sent to the pathology laboratory. Cytopathologists of the hospital specializing in thyroid pathology interpreted the smears. During the study period, the cytology reports were classified as benign, indeterminate, suspicious for carcinoma, malignant, or inadequate. Histology was performed if cytology was indeterminate or suggestive of malignancy.

TIRADS classification algorithm from Russ classification [13] is showed in Figure 1.

\subsection{Data Collection and Analysis}

A standardized form was used to collect data. Sensitivity, specificity, positive predictive value (PPV) and negative predictive value (NPV) were calculated for each of the "major" US features that highly suggest malignancy (irregular contours, taller-than-wide, presence of microcalcifications, marked hypoechogenicity) according to Kwak JY et al. [5] and Kim E-Y et al. [6]. Risk estimates (odds ratio) were calculated and presented using 95\% confidence interval (CI) statistic. The risk of malignancy of each TIRADS category was determined. Symmetric measures (ordinal by ordinal gamma statistic, Spearman correlation and Pearson's rho) were used to assess the strength of the linear relationship between the benign and 


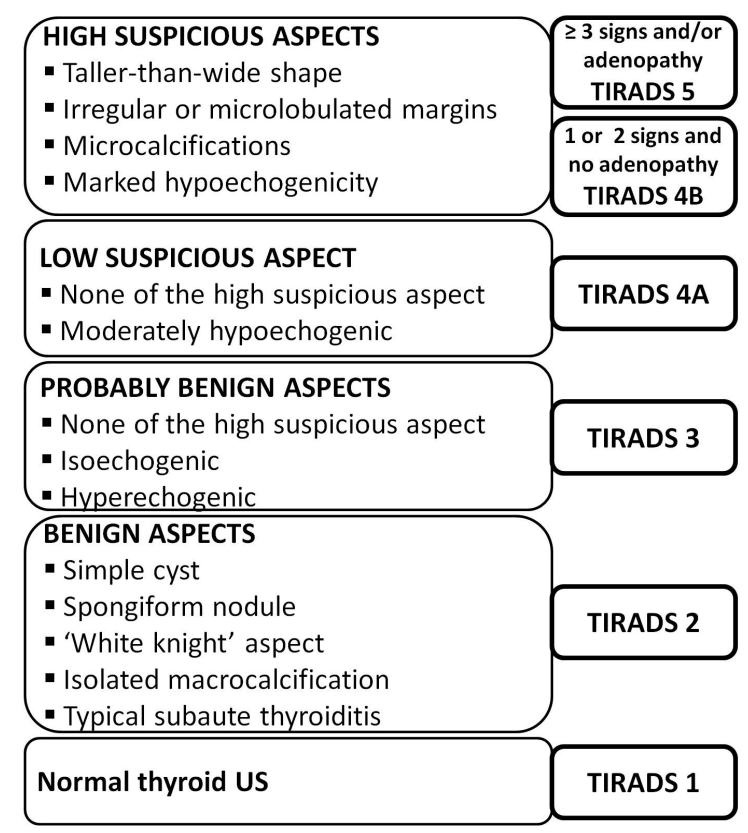

Figure 1. TIRADS classification algorithm [13].

the malignant groups with respect to the TIRADS categories. The threshold for statistical significance was set at 0.05 . All statistical analysis was performed using the software IBM SPSS 20.0 (SPSS Inc, Chicago, USA).

\section{Results}

A total of 430 records of patients who fulfilled the inclusion criteria were selected for the study. Twenty-three out of 430 (5.35\%) nodules were histologically proven to be malignant.

\subsection{TIRADS Categories, Cytology Results and Risk of Malignancy}

The US features of each thyroid nodule were characterized and classified into different TIRADS categories, as shown in Figure 2. The TIRADS 3 category was dominant, accounting for 226 cases (52.6\%).

The different TIRADS categories were confronted with the results of pathology and the risk of malignancy was calculated (Table 1). The risk of malignancy was found to increase from TIRADS 3 to 5. Computed symmetric statistics for the strength of association between benign and malignant cytology results in the different TIRADS categories were found to be statistically significant $(\mathrm{p}<0.001)$.

Combining TIRADS 2, 3 and $4 \mathrm{~A}$ as probably benign US findings, and TIRADS $4 \mathrm{~B}$ and 5 as probably malignant US findings as shown in Table 2, the sensitivity, specificity, positive predictive value and negative predictive value were respectively $98.03 \%, 52.17 \%, 97.32 \%$ and $60 \%$. The overall accuracy of ultrasound was $95.58 \%$.

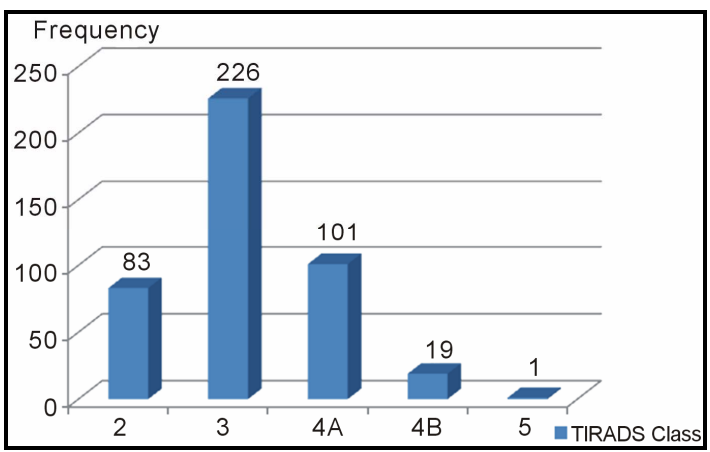

Figure 2. TIRADS categories of the study population.

Table 1. TIRADS categories and risk of malignancy.

\begin{tabular}{ccccc}
\hline \multirow{2}{*}{$\begin{array}{c}\text { TIRADS } \\
\text { category }\end{array}$} & \multicolumn{2}{c}{ Pathology } & Total & $\begin{array}{c}\text { Risk of malignancy } \\
(\%)\end{array}$ \\
\cline { 2 - 3 } & Benign & Malignant & & 0 \\
\hline TIRADS 2 & 83 & 0 & 83 & 2.2 \\
TIRADS 3 & 221 & 5 & 226 & 5.9 \\
TIRADS 4A & 95 & 6 & 101 & 57.9 \\
TIRADS 4B & 8 & 11 & 19 & 100 \\
TIRADS 5 & 0 & 1 & 1 & - \\
Total & 407 & 23 & 430 & - \\
\hline
\end{tabular}

Symmetric measures: Gamma statistic $=0.85(\mathrm{p}<0.001)$; Spearman correlation $=0.30(\mathrm{p}<0.001)$; Pearson's $\mathrm{R}=0.37(\mathrm{p}<0.001)$.

Table 2. TIRADS categories and diagnostic performance of US.

\begin{tabular}{cccc}
\hline \multirow{2}{*}{ TIRADS category } & \multicolumn{2}{c}{ Pathology } & \multirow{2}{*}{ Total } \\
\cline { 2 - 3 } & Benign & Malignant & \\
\hline TIRADS 2, 3, 4A & 399 & 11 & 410 \\
TIRADS 4B, 5 & 8 & 12 & 20 \\
Total & 407 & 23 & 430 \\
\hline
\end{tabular}

\section{2. "Major" Ultrasound Findings}

The "major" US features suggestive of malignancy were analyzed with respect to TIRADS categories. Sensitivity, specificity, positive predictive value, negative predictive value and odds ratio were calculated for each feature. Tables 3 and 4 show the different statistical analysis of the major US features with respect to cytology/histology results, and their respective performance.

A summary of the major US features suggestive of malignancy are presented in Table 4 alongside their respective performance.

\section{Discussion}

The acronym TIRADS seems to have come to stay. It harmonizes the reporting of thyroid US findings in a very simply way that facilitates comprehension across different specialties. For any such classification system to be 
Table 3. Major US features and pathology results.

\begin{tabular}{ccccc}
\hline \multirow{2}{*}{ Major ultrasound features } & \multicolumn{2}{c}{ Pathology } & \multirow{2}{*}{ Total } \\
\cline { 2 - 4 } & & Benign & Malignant & \\
\hline \multirow{2}{*}{ Irregular margins } & Present & 2 & 8 & 10 \\
& Absent & 405 & 15 & 420 \\
\multirow{2}{*}{$\begin{array}{c}\text { Taller-than-wide } \\
\text { shape }\end{array}$} & Present & 0 & 1 & 1 \\
\multirow{2}{*}{$\begin{array}{c}\text { Microcalcification } \\
\text { Marked }\end{array}$} & Absent & 407 & 22 & 429 \\
& Present & 5 & 7 & 12 \\
hypoechogenicity & Present & 2 & 3 & 418 \\
\hline
\end{tabular}

Irregular margins: odds ratio for benign cytology: 0.21 (95\%CI: 0.06 0.72). Odds ratio for malignant cytology: 22.40 (95\%CI: 12.47 - 40.23). Taller-than-wide shape: odds ratio for malignant cytology: 19.50 (95\%CI: 12.98 - 29.25). Microcalcification: odds ratio for benign cytology: 0.43 (95\%CI: 0.22 - 0.85). Odds ratio for cytology: 15.24 (95\%CI: 7.74 - 30.02). Marked hypoechogenicity: odds ratio for benign cytology: 0.42 (95\%CI: 0.14 - 1.23). Odds ratio for malignant cytology: 12.75 (95\%CI: 5.54 29.35).

Table 4. Summary of the statistical performance of the major US features.

\begin{tabular}{cccccc}
\hline US feature & Se (\%) & Sp (\%) & $\begin{array}{c}\text { PPV } \\
(\%)\end{array}$ & $\begin{array}{c}\text { NPV } \\
(\%)\end{array}$ & OR \\
\hline $\begin{array}{c}\text { Irregular contours } \\
\text { Taller-than-wide } \\
\text { shape }\end{array}$ & 34.78 & 99.51 & 80 & 96.43 & 22.40 \\
$\begin{array}{c}\text { Microcalcification } \\
\begin{array}{c}\text { Marked } \\
\text { hypoechogenicity }\end{array}\end{array}$ & 13.04 & 100 & 100 & 94.87 & 19.50 \\
\hline
\end{tabular}

Se = Sensitivity; Sp = Specificity; PPV = Positive Predictive Value; NPV = Negative Predictive Value; OR = Odd Ratio.

useful for routine clinical practice, it should be simple to use, reproducible and very reliable.

Thyroid cancer is a relatively rare entity, with an estimated prevalence of about $5 \%[4,5,14]$. The proportion of malignant thyroid nodules obtained in this study was similar to this value. A high accuracy of any classification in predicting malignant thyroid lesions will be particularly of help in resource-limited settings where pathological analysis is not routinely performed even when confronted with some suspiciously malignant lesions. The diagnostic accuracy of US in this study exceeded to that obtained by Moon et al. [15] in 2002.

From our results, the risk of malignancy significantly increased from TIRADS 3 to 5 . This was zero for TIRADS 2, and would be expected to be so since TIRADS 2 is considered ultrasonographically as a typically benign lesion. In his work, Horvath suggested a malignant risk of less than $5 \%$ for TIRADS 3, $5 \%$ to $10 \%$ for TIRADS $4 \mathrm{~A}, 10 \%$ to $80 \%$ for TIRADS $4 \mathrm{~B}$ and greater than $80 \%$ for TIRADS 5 [9]. Our findings are within this range suggested by Horvath and similar to that obtained by Russ et al. [12]. This is capital in risk stratification for malignancy of thyroid nodules. So if properly classified on US the probability of a particular nodule being malignant can be inferred from the TIRADS category with a certain level of confidence and appropriate measures for management can be initiated.

Most cancers were found in the TIRADS 3, 4A and 4B categories. We can infer from this that most cancers will have US features that may seem probably benign ultrasonographically, or have features that mimic a low or a high suspicion for malignancy. So very few cases of malignancy will have the very typical ultrasound features that are consistent with malignancy. This further justifies the advocacy for FNAB when lesions are not typically benign ultrasonographically.

The presence of some US features had earlier been described as highly suspicious for malignancy, and they include marked hypoechogenicity, taller-than-wide shape, irregular contours and the presence of calcifications $[5,6$, 12]. In our study, these features were found to be highly suspicious for malignancy as can be seen from the odds ratios, sensitivities, specificities, PPV and NPV. However we did not assess the probabilities of malignancy of associated features, which was found to increase in a previous study [5]. In one study Hong YJ et al. [16] concluded that the three sonographic features that are meaningful findings in the diagnosis of thyroid malignancy were the presence of microcalcifications, marked hypoechogenecity and a taller-than-wide shape. In a multicentre Korean retrospective study, the US features that were statistically significant for malignant thyroid nodules were hypoechogenicity, marked hypoechogenicity, nonparallel orientation, microlobulated or speculated margin, ill-defined margins and the presence of micro-calcifications [17]. In the latest study, 7.3\% of malignant nodules did not have suspicious-malignant features on US.

This study has not been void of limitations, which include retrolective data collection and the fact that histology was not available for all of the thyroid nodules, as those with a benign cytology were not operated for ethical reasons. However, this can be compensated by the high NPV of cytology.

\section{Conclusion}

Russ' modified TIRADS classification is reliable in predicting thyroid malignancy. We therefore advocate for further studies in the same light for more evidence and the validation of a classification system for the thyroid gland that will be simple to use, reliable, reproducible and facilitate communication across different clinical specialties. 


\section{Acknowledgements}

The authors wish to thank the team of the Pathology Department of the Centre Hospitalier de Lagny, Marne La Vallée (France) for helping with cytology/histology records of patients in this study.

\section{REFERENCES}

[1] L. E. Koike, S. Noguchi, H. Yamashita, T. Murakami, A. Ohshima, H. Kawamoto and H. Yamashita, "Ultrasonographic Characteristics of Thyroid Nodules: Prediction of Malignancy,” Archives of Surgery, Vol. 136, No. 3, 2001, pp. 334-337. doi:10.1001/archsurg.136.3.334

[2] E. Papini, R. Guglielmi, A. Bianchini, A. Crescenzi, S. Taccogna, F. Nardi, C. Panunzi, R. Rinaldi, V. Toscano and C. M. Pacella, "Risk of Malignancy in Nonpalpable Thyroid Nodules: Predictive Value of Ultrasound and Color-Doppler Features," The Journal of Clinical Endocrinology \& Metabolism, Vol. 87, No. 5, 2002, pp. 19411946. doi:10.1210/jc.87.5.1941

[3] J. K. Hoang, W. K. Lee, M. Lee, D. Johnson and S. Farrell, "US Features of Thyroid Malignancy: Pearls and Pitfalls,” Radiographics, Vol. 27, No. 3, 2007, pp. 847860. doi:10.1148/rg.273065038

[4] K. M. Kim, J. B. Park, S. J. Kang and K. S. Bae, "Ultrasonographic Guideline for Thyroid Nodules Cytology: Single Institute Experience,” Journal of the Korean Surgical Society, Vol. 84, No. 2, 2013, pp. 73-79. doi:10.4174/jkss.2013.84.2.73

[5] J. Y. Kwak, K. H. Han, J. H. Yoon, H. J. Moon, E. J. Son, S. H. Park, et al., "Thyroid Imaging Reporting and Data System for Ultrasound Features of Nodules: A Step in Establishing Better Stratification of Cancer Risk,” Radiology, Vol. 260, No. 3, 2011, pp. 892-899. doi:10.1148/radiol.11110206

[6] E. K. Kim, C. S. Park, W. Y. Chung, K. K. Oh, D. I. Kim, et al., "New Sonographic Criteria for Recommending Fine-Needle Aspiration Biopsy of Nonpalpable Solid Nodules of the Thyroid," American Journal of Roentgenology, Vol. 178, No. 3, 2002, pp. 687-691. doi:10.2214/ajr.178.3.1780687

[7] M. C. Frates, C. B. Benson, J. W. Charboneau, E. S. Cibas, O. H. Clark, et al., "Management of Thyroid Nodules Detected at US: Society of Radiologists in Ultrasound Consensus Conference Statement,” Radiology, Vol. 237, No. 3, 2005, pp. 794-800. doi:10.1148/radiol.2373050220
[8] C. Capelli, M. Castellano, I. Pirola, D. Cumetti, B. Agosti, E. Gandossi and E. Agabiti Rosei, "The Predictive Value of Ultrasound Findings in the Management of Thyroid Nodules," QJM: An International Journal of Medicine, Vol. 100, No. 1, 2007, pp. 29-35. doi:10.1093/qjmed/hcl121

[9] E. Horvath, S. Majlis, R. Rossi, C. Franco, J. P. Niedmann, et al., "An Ultrasonogram Reporting System for Thyroid Nodules Stratifying Cancer Risk for Clinical Management," The Journal of Clinical Endocrinology \& Metabolism, Vol. 95, No. 5, 2009, pp. 1748-1751. doi:10.1210/jc.2008-1724

[10] American College of Radiology, "Breast Imaging Reporting and Data System. Breast Imaging Atlas,” 4th Edition, American College of Radiology, Reston, 2003.

[11] J. Y. Park, H. J. Lee, H. W. Jang, H. K. Kim, J. H. Hi, et al., "A Proposal for a Thyroid Imaging Reporting and Data System for Ultrasound Features of Thyroid Carcinoma,” Thyroid, Vol. 19, No. 11, 2009, pp. 1257-1264. doi:10.1089/thy.2008.0021

[12] G. Russ, C. Bigorgne, B. Royer, A. Rouxel and M. Bienvenu-Perrard, "The Thyroid Imaging Reporting and Data System (TIRADS) for Ultrasound of the Thyroid,” Journal of Radiology, Vol. 92, No. 7-8, 2011, pp. 701-713. doi:10.1016/j.jradio.2011.03.022

[13] G. Russ, "Thyroid Imaging and Reporting Database System,” 2013. www.tirads.com.

[14] D. S. Dean and H. Gharib, "Epidemiology of Thyroid Nodules," Best Practice \& Research: Clinical Endocrinology \& Metabolism, Vol. 22, No. 6, 2008, pp. 901-911. doi:10.1016/j.beem.2008.09.019

[15] H. J. Moon, J. M. Sung, E. K. Kim, J. H. Yoon, J. H. Youk and J. Y. Kwak, "Diagnostic Performance of GrayScale US and Elastography in Solid Thyroid Nodules," Radiology, Vol. 262, No. 3, 2012, pp. 1002-1013. doi:10.1148/radiol.11110839

[16] Y. J. Hong, E. J. Son, E. K. Kim, J. Y. Kwak, S. W. Hong and H. S. Chang, "Positive Predictive Values of Sonographic Features of Solid Thyroid Nodule," Clinical Imaging, Vol. 34, No. 2, 2010, pp. 127-133. doi:10.1016/j.clinimag.2008.10.034

[17] J. Y. Kwak, I. Jung, J. H. Baek, S. M. Baek, N. Choi, Y. J. Choi, et al., "Image Reporting and Characterization System for Ultrasound Features of Thyroid Nodules: multicentrickorean Retrospective Study," Korean Journal of Radiology, Vol. 14, No. 1, 2013, pp. 110-117. doi:10.3348/kjr.2013.14.1.110. 